\title{
Long-Term Probucol Treatment Prevents Secondary Cardiovascular Events: a Cohort Study of Patients with Heterozygous Familial Hypercholesterolemia in Japan
}

\author{
Shizuya Yamashita ${ }^{1}$, Hideaki Bujo ${ }^{2}$, Hidenori Arai $^{3}$, Mariko Harada-Shiba ${ }^{4}$, Shigeyuki Matsui ${ }^{5}$, \\ Masanori Fukushima ${ }^{6}$, Yasushi Saito ${ }^{7}$, Toru Kita ${ }^{8}$, and Yuji Matsuzawa ${ }^{9}$ \\ ${ }^{1}$ Department of Cardiovascular Medicine, Osaka University Graduate School of Medicine, Osaka, Japan \\ ${ }^{2}$ Department of Genome Research and Clinical Application, Chiba University Graduate School of Medicine, Chiba, Japan \\ ${ }^{3}$ Department of Geriatric Medicine, Kyoto University Graduate School of Medicine, Kyoto, Japan \\ ${ }^{4}$ Department of Bioscience, National Cardiovascular Center Research Institute, Osaka, Japan \\ ${ }^{5}$ Department of Pharmacoepidemiology, School of Public Health, Kyoto University, Kyoto, Japan \\ ${ }^{6}$ Translational Research Center, Kyoto University Hospital, Kyoto University Graduate School of Medicine, Kyoto, Japan \\ ${ }^{7}$ Department of Clinical Cell Biology, Chiba University Graduate School of Medicine, Chiba, Japan \\ ${ }^{8}$ Department of Cardiovascular Medicine, Kyoto University Graduate School of Medicine, Kyoto, Japan \\ ${ }^{9}$ Department of Internal Medicine, Sumitomo Hospital, Osaka, Japan
}

Aim: The POSITIVE study assessed whether long-term treatment with probucol, a potent anti-oxidant and cholesteryl ester transfer protein (CETP) activator, is associated with a lowered risk of cardiovascular events in a very high-risk population: familial hypercholesterolemia (FH).

Methods: The study cohort included 410 patients with heterozygous FH, diagnosed between 1984 and 1999 by cardiovascular and metabolic experts at fifteen centers. Traceable patients were screened using predefined eligibility criteria. The primary outcome measure for comparison between probucol exposure and non-exposure was the time to the first cardiovascular event involving hospitalization.

Results: Analysis revealed significant differences in baseline characteristics and follow-up treatment between exposure and non-exposure. An observed indication bias was the use of probucol in more severe FH at diagnosis, both for primary and secondary prevention. When the multivariate Cox regression procedure was used after adjustment for possible confounding factors, probucol lowered the risk (hazard ratio $[\mathrm{HR}], 0.13 ; 95 \%$ confidence interval $[\mathrm{CI}], 0.05-0.34$ ) in secondary prevention $(n=74)$ and was statistically significant $(p<0.001)$, although not significant $(\mathrm{HR}, 1.5 ; 95 \% \mathrm{CI}$, $0.48-4.67 ; p=0.49)$ in primary prevention $(n=233)$. Safety assessment found no specific difference between exposure and non-exposure.

Conclusion: Long-term probucol treatment may prevent secondary attack in a higher cardiovascular risk population of heterozygous $\mathrm{FH}$.

J Atheroscler Thromb, 2008; 15:292-303.

Key words; Atherosclerosis, Antioxidants, CETP activator, Dyslipidemia

\section{Introduction}

Cardiovascular $(\mathrm{CV})$ diseases, including coronary

Address for correspondence: Shizuya Yamashita, Department of Cardiovascular Medicine, Osaka University Graduate School of Medicine, 2-2 Yamadaoka, Suita, Osaka 565-0871, Japan

E-mail: shizu@imed2.med.osaka-u.ac.jp

Received: July 17, 2008

Accepted for publication: September 19, 2008 heart disease and stroke, are the leading cause of death in Japan. Prevention of fatal CV events is therefore the final goal as well as the rationale of cholesterol-lowering therapy.

Probucol, a conventional cholesterol-lowering drug, originated with the report by Barnhart in $1970^{11}$. The drug has been used clinically in Japan since 1985. Nearly 60,000 Japanese patients still take probucol; western countries discontinued probucol use after 
the original manufacturer's withdrawal notice to the United States FDA in 1995 after 18 year's use of the drug. Probucol's cholesterol-lowering mechanism has not yet been clearly established, but it is thought to increase catabolic excretion of cholesterol into bile ${ }^{2)}$. Later studies ${ }^{3-5)}$ have described new mechanisms of probucol, including anti-atherogenic and anti-oxidant actions. Another controversial and anti-atherogenic feature of probucol is its paradoxical effect of lowering high-density lipoprotein cholesterol (HDL-C). This action reflects, most likely, its molecular mechanisms: promoting cholesterol efflux, and enhancing reverse cholesterol transport by activation of cholesteryl ester transfer protein (CETP ${ }^{6-8)}$ and class B type 1 scavenger receptor ${ }^{9,10)}$. Matsuzawa and his colleagues reported an observed close correlation between the extent of regression in Achilles' tendon xanthoma and probucol-induced decrease in HDL-C levels in patients with familial hypercholesterolemia $(\mathrm{FH})^{11)}$.

No large-scale, randomized, double blind comparative study has been conducted to justify the use of probucol in the prevention of $\mathrm{CV}$ events or diseases. however, clinical studies as well as pre-clinical data have been accumulating evidence of the clinical worth of probucol in arteriosclerotic diseases. Numerous clinical results, including a reduction in Achilles' tendon xanthoma thickness after long-term treatment for $\mathrm{FH}^{12,13)}$, reduced rates of restenosis after angioplasty ${ }^{14-16)}$, and a decrease in carotid artery intimamedia thickness ${ }^{17,18)}$ support the therapeutic and preventative effects of probucol on arteriosclerotic lesions and plaque. To evaluate the risk and benefit of longterm probucol treatment, we conducted a cohort study to determine whether probucol treatment is associated with the risk reduction of CV events in patients with heterozygous $\mathrm{FH}$, a very high-risk population.

\section{Methods}

\section{Study Cohort}

We registered patients with $\mathrm{FH}$ who received treatment between January 1, 1984 and December 31, 1999 at 15 centers specializing in CV and metabolic diseases, including $\mathrm{FH}$, nationwide. Patients were traceable by medical recend and met the diagnostic criteria for heterozygous FH under the Japan Atherosclerosis Society Guidelines (2002) for the Diagnosis and Treatment of Atherosclerotic CV Diseases ${ }^{19)}$. Definite heterozygous $\mathrm{FH}$ was defined as having at least two of the major features: total cholesterol (TC) of $260 \mathrm{mg} / \mathrm{dL}$ and above; tendon xanthoma or xanthoma tuberosum; reduced or abnormal receptor activity noted by LDL receptor analysis. Probable heterozy- gous $\mathrm{FH}$ was defined as having at least one each of the major (as above) and minor features: palpebral xanthoma; arcus juvenilis ( $<50$ years); juvenile $(<50$ years) ischemic heart disease. For other eligibility criteria, we excluded patients with possible homozygous FH or with severe ventricular arrhythmias (polymorphic premature ventricular contractions). Possible homozygous $\mathrm{FH}$ was defined as having any one of the clinical features: defect of homozygous or hetero-polymeric LDL receptors confirmed by gene analysis; no LDLR activity observed by receptor analysis, severe elevation of plasma TC higher than $500 \mathrm{mg} / \mathrm{dL}$; xanthoma or atherosclerotic vascular lesions including symptoms of juvenile ischemic heart disease; hypercholesterolemia confirmed in both parents; history of ischemic heart disease confirmed in both parents; or poor response to any 3-hydroxy-3methyl-glutarylcoenzyme A reductase inhibitor (statin).

During the study period between June, 2004 and September, 2005, we collected anonymous case report forms with the patients' baseline data, including medical history, findings at clinical examination, medication data, and laboratory data. The investigators transcribed the data on to case report forms (identified by a code) from the stored medical charts of the patients. The observation period was the period for which each patient's clinical course could be traced. The longest observation period exceeded 20 years for patients on stable doses of probucol.

We required a sample size of 200 in both the probucol exposure and non-exposure groups, supposing a difference of $10 \%$ in the incidence of $\mathrm{CV}$ events for 5 years $(15 \%$ in exposure and $25 \%$ in non-exposure). A least 400 subjects were needed to detect the difference with $80 \%$ power and a type I error of $5 \%$ at the $5 \%$ significance level with two-sided log-rank test based on normal approximation. The study protocol was approved through the process of ethics committee or institutional review board at each center.

\section{Definitions and Endpoints}

The primary outcome measure was the time to the first CV event, defined as acute myocardial infarction (MI), angina pectoris (AP), heart failure (HF), stroke, transient ischemic attack (TIA) or arteriosclerotic peripheral artery diseases (PAD) leading to hospitalization or death as well as sudden death within 24 hours of an observed intrinsic event. The obtained baseline data at the first visit of each patient included demographic characteristics: sex, date of diagnosis at the participant medical center, age, height, weight, and habits of smoking and drinking. Body mass index (BMI) was calculated as weight in kilograms divided 
by the square of height in meters. The other collected characteristic factors at diagnosis were the presence of xanthoma and its location, prior CV event, onset date if any prior $\mathrm{CV}$ event, treatment for the event, and other possible risk factors for $\mathrm{CV}$ events, including the presence of hypertension, diabetes, ventricular arrhythmia, and PAD. We collected data on cholesterol-lowering therapy (with or without probucol) and other concomitant therapy with anti-platelet, antihypertensive or diabetic drugs. Dates of drug initiation, discontinuation, re-administration, and termination were entered as elemental information. Treatment period was defined as the length from initiation until medication termination, or until the occurrence of the defined CV event, whichever came first. A lipid profile of TC, triglyceride (TG), low-density lipoprotein cholesterol (LDL-C) and HDL-C, blood pressure, level of fasting blood sugar (FBS), hemoglobin $A_{1 c}\left(\mathrm{HbA}_{1 \mathrm{c}}\right)$, and thickness of tendon xanthoma in both feet were variables of interest, seen as potential predictors of CV events. We obtained measurements of those variables on a yearly basis after each patient was diagnosed. LDL-C levels were calculated from TC and HDL-C measurements with the Friedewald formula in TG $<400 \mathrm{mg} / \mathrm{dL}$. For TG of $400 \mathrm{mg} / \mathrm{dL}$ and more than $400 \mathrm{mg} / \mathrm{dL}$, the expression of $0.16 \mathrm{X} \mathrm{TG}$ was applied in stead of $0.2 \mathrm{X} \mathrm{TG}^{20)}$. Most patients had fasted compliantly at periodic checkups of their lipid levels. We set a follow-up period of 10 years for the measurements.

\section{Statistical Analyses}

The primary objective of analysis was a comparison between probucol exposure and non-exposure to evaluate whether treatment with probucol $(500 \mathrm{mg}$ to $1,000 \mathrm{mg}$ daily) for $\mathrm{FH}$ provided $\mathrm{CV}$ benefits. The analysis was based on intent-to-treat principles. The secondary objective was to assess whether changes in the lipid profile after probucol treatment predicted $\mathrm{CV}$ events in the cohort. Event-free survival, defined as the time from diagnosis to the first CV event, was determined as a response variable. Statistical analysis was performed to evaluate clinical outcomes separately for secondary and primary prevention groups; that is, patients with or without a history of CV events at diagnosis.

Baseline characteristics of each group were explored to detect risk factors for CV events because potential confounders, including indication bias, were anticipated. For baseline comparison, Wilcoxon's rank sum test and Fisher's exact test were used for continuous variables and categorical variables respectively. For detection of risk factors, univariate Cox proportional hazards regression with a baseline variable as covariate was used as a screening step to determine the relationship with CV events. Variables that achieved significance at the level of $20 \%$ in univariate analysis were subsequently included in a multivariate Cox proportional hazards regression using backward variable selection. Variables proving significant at the $10 \% \mathrm{sig}$ nificance level were selected as risk factors to be adjusted. Consequently, probucol treatment effect was evaluated using the multivariate Cox model with adjustment for the selected baseline variables. Finally, the other observed treatment factors: cholesterol-lowering drugs other than probucol, LDL-apheresis, anti-platelet drugs, anti-hypertensive drugs, and diabetic drugs were entered into that model to assess their effects.

For the association between changes in lipid profile after probucol treatment and the risk of $\mathrm{CV}$ events, pre-treatment values of TG, LDL-C, HDL-C as well as TC, and each lipid reduction ratio after treatment were used as covariates. Multivariate analyses of time from probucol start to the first CV event used multivariate Cox's proportional hazards models. Statistical analysis was peerformed with SAS version 8.2.

\section{Results}

\section{Patient Characteristics}

We collected data from the medical records of 541 patients, and excluded the data of 131 patients that did not meet eligibility predefined in the protocol.

The flow diagram (Fig. 1) gives reasons for the exclusion. A substantial fraction of probucol-exposed patients, $80.0 \%$ and $93.2 \%$, took probucol within two years after diagnosis for in primary and secondary prevention groups, respectively. Baseline characteristics at diagnosis are given for each group (Table 1, 2). The secondary prevention group (Table 2) had prior diseases of AP, MI, stroke, HF, and TIA. This group was found to have significant higher proportions of men $(60.2 \%, p<0.01)$, smokers $(50.0 \%, p<0.01)$, hypertension $(40.9 \%, p<0.001)$ diabetes $(15.9 \%, p=$ 0.02 ), and older median age (52 years, $p=0.01$ ) than the primary prevention group. Moreover, the group tended to have hypo-HDL cholesterolemia of median $42(20-90) \mathrm{mg} / \mathrm{dL}$, and to receive combined treatments with anti-platelet drugs (56.8\%), anti-hypertensive drugs (53.4\%), and LDL-apheresis (14.8\%).

Comparison between probucol-exposed and nonexposed groups revealed significant differences in some baseline characteristics and treatments, which showed a confounding indication that patients with more severe $\mathrm{FH}$ took probucol. For baseline characteristics, the exposed group for primary prevention had more 


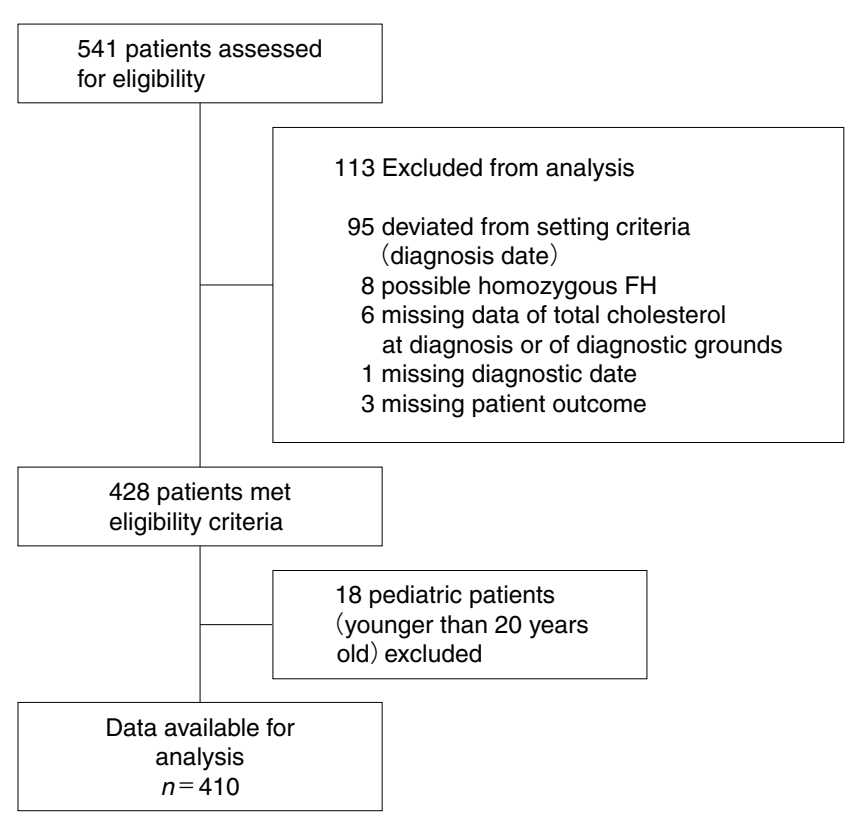

Fig. 1. Patient Flowchart.

We collected data from the medical records of 541 patients, and excluded the data of 131 patients who did not meet the eligibility predefined in the protocol. The flow diagram gives reasons for the exclusion.

palpebral xanthoma $(13.4 \%, p=0.05)$, thicker median measurement of tendon xanthoma $(12.5 \mathrm{~mm}, p<0.01)$, higher median $\mathrm{HbA}_{1 \mathrm{c}}(5.8 \%, p=0.03)$, and more use of antihypertensive drugs $(25.3 \%, p<0.01)$. Their lipid profile was more severe with a higher median baseline TC $(325 \mathrm{mg} / \mathrm{dL}, p=0.001)$, a higher median LDL-C level $(253 \mathrm{mg} / \mathrm{dL}, p<0.001)$, and a lower HDL-C level $(47 \mathrm{mg} / \mathrm{dL}, p<0.001)$ than the unexposed group. The exposed group for secondary prevention had a higher prevalence of post-MI (44.6\%, $p<0.01)$ than the unexposed group. Observed medications were also significantly different between the exposed and unexposed groups. The exposed group used anti-hypertensive drugs concomitantly at a higher rate $(25.3 \%$ vs. $11.2 \%, p<0.01)$ for primary prevention.

Descriptive analysis of baseline characteristics and treatments during observation implies that in both primary and secondary prevention, the exposed groups tended to include patients with more severe $\mathrm{FH}$ at diagnosis. Arguably, patients considered more severe at diagnosis would receive more intensive treatment, including probucol.

\section{Outcomes}

We present the absolute number of $\mathrm{CV}$ events requiring hospitalization by prevention group with details of the events (Table 3). The incidence of CV events without consideration of confounding factors was $11.6 \%$ in the exposed group and $4.5 \%$ in the unexposed group for primary prevention. For secondary prevention, the incidence was $27.0 \%$ in the exposed group and $64.3 \%$ in the unexposed group. The event-free survival curve of the secondary prevention group is given (Fig. 2).

To identify risk factors for CV events, we determined the relationship between the incidence and every baseline variable using univariate Cox regression at a significant level of $20 \%$. Variables proving significant at the $10 \%$ significance level in multivariate Cox regression were selected as risk factors to be adjusted. We estimated the effect of treatment after adjusting the selected risk factors. We calculated hazard ratios (HRs) with $95 \%$ confidence interval (CI) for binary variables, $\mathrm{BMI} \geq 25$ vs $\mathrm{BMI}<25$, drinking vs no drinking, for example, and the indicated HRs corresponded to a 1 standard deviation increase for continuous variables, including TC. Estimated results are given (Table 4).

In the primary prevention group, significant variables were $\mathrm{BMI} \geq 25$ (HR 1.86, 95\% CI 0.87-3.98; $p=0.11$ ), drinking (HR 2.17, 95\% CI 1.02-4.63; $p=0.05$ ), tendon xanthoma (HR 2.17, 95\% CI 0.766.23 ; $p=0.15)$, prior diseases other than $\mathrm{CV}$ events (HR 1.87, 95\% CI 0.87-3.99; $p=0.11$ ), PAD (HR 5.23, 95\% CI 0.70-39.2; $p=0.11$ ), diabetes (HR 2.27, 95\% CI $0.79-6.50 ; p=0.13)$, TC (HR 1.37, 95\% CI $0.99-1.89 ; p=0.06)$, HDL-C (HR 0.75, 95\% CI $0.50-1.12, p=0.16$ ), SBP (HR 1.48, 95\% CI $1.00-$ $2.18 ; p=0.05)$, and the thickness of tendon xanthoma (HR 1.50, 95\% CI 1.06-2.14; $p=0.02$ ). Three of these variables, drinking, TC, and PAD were selected for adjustment at the $10 \%$ significance level as a result of a multivariate Cox regression with backward variable selection. After adjustment for these three baseline variables, we found no significant effect by probucol at the 5\% significant level. The estimated hazard ratio of probucol use for CV events was 1.50 (95\% CI $0.48-4.67 ; p=0.49)$.

In the secondary prevention group, significance variables were drinking (HR 1.74, 95\% CI 0.80-3.79; $p=0.17$ ), presence of palpebral xanthoma (HR 5.34, 95\% CI 2.26-12.61, $p<0.001$ ), TIA (HR 4.16, 95\% CI $0.54-32.21 ; p=0.17)$, history of coronary artery bypass graft (HR $0.31,95 \%$ CI $0.11-0.90 ; p=0.03$ ), hypertension (HR $0.58,95 \%$ CI $0.26-1.28 ; p=0.18$ ), diabetes (HR 2.89, 95\% CI 1.30-6.42; $p<0.01$ ), and fasting blood sugar (HR 1.31, 95\% CI 0.91-1.89; $p=$ $0.15)$. Two of these variables, palpebral xanthoma and diabetes, were selected for adjustment at the $10 \%$ sig- 
Table 1. Baseline characteristics of patients in primary prevention group

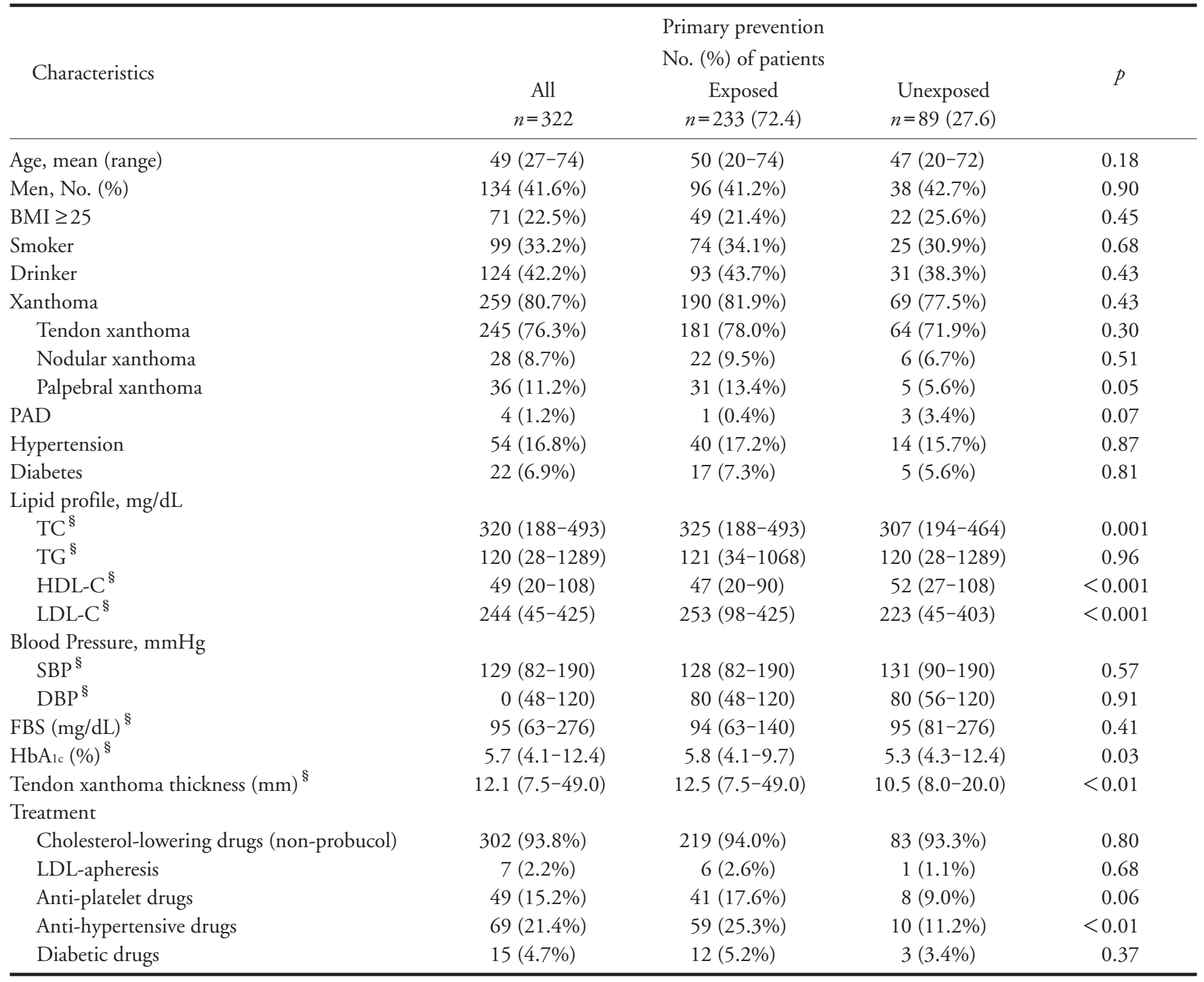

'Continuous variables compared by Wilcoxon's rank sum test, distribution of categorical variables by Fisher's exact test. ${ }^{\S}$ Data are median (range). All data are number (\%) unless otherwise indicated. Each percentage shown is related to the total number with measurement data. BMI, body mass index; PAD, peripheral artery disease; TC, total cholesterol; TG, triglyceride; HDL-C, high-density lipoprotein cholesterol; LDL-C, low-density lipoprotein cholesterol; SBP, systolic blood pressure; DBP, diastolic blood pressure; FBS, fasting blood sugar; HbA1c, hemoglobin A1c. LDL-C was calculated with the Friedewald formula.

nificance level as a result of multivariate Cox regression analysis using a backward variable selection. After adjustment for these two baseline variables, the hazard ratio of probucol use for $\mathrm{CV}$ events was estimated to be 0.13 (95\% CI $0.05-0.34)$ and significant $(p<$ $0.001)$. In sensitivity analyses, we also obtained similar estimation results on probucol for various sets of baseline covariates for adjustment.

The lipid levels of TC, LDL-C and HDL-C were lowered after probucol treatment both in primary and secondary prevention. In the primary prevention group, the median (range) levels of TC, TG, LDL-C and HDL-C closest to before treatment were respectively 305 (165-493), 119 (35-1068), 228 (107-425) and $48(25-96) \mathrm{mg} / \mathrm{dL}$, and those at 10 -year treatment were, respectively, 222 (141-371), 94 (43-335), 157 (91-311) and 39 (17-81) $\mathrm{mg} / \mathrm{dL}$. In the secondary prevention, the median levels of TC, TG, LDL-C and HDL-C closest to before atment were, respectively, 320 (191-469), 129 (37-636), 240 (117-381) and $44(24-90) \mathrm{mg} / \mathrm{dL}$, and those at 10-year treatment were, respectively, 211(135-305), 71 (48-475), 
Table 2. Baseline characteristics of patients in secondary prevention group

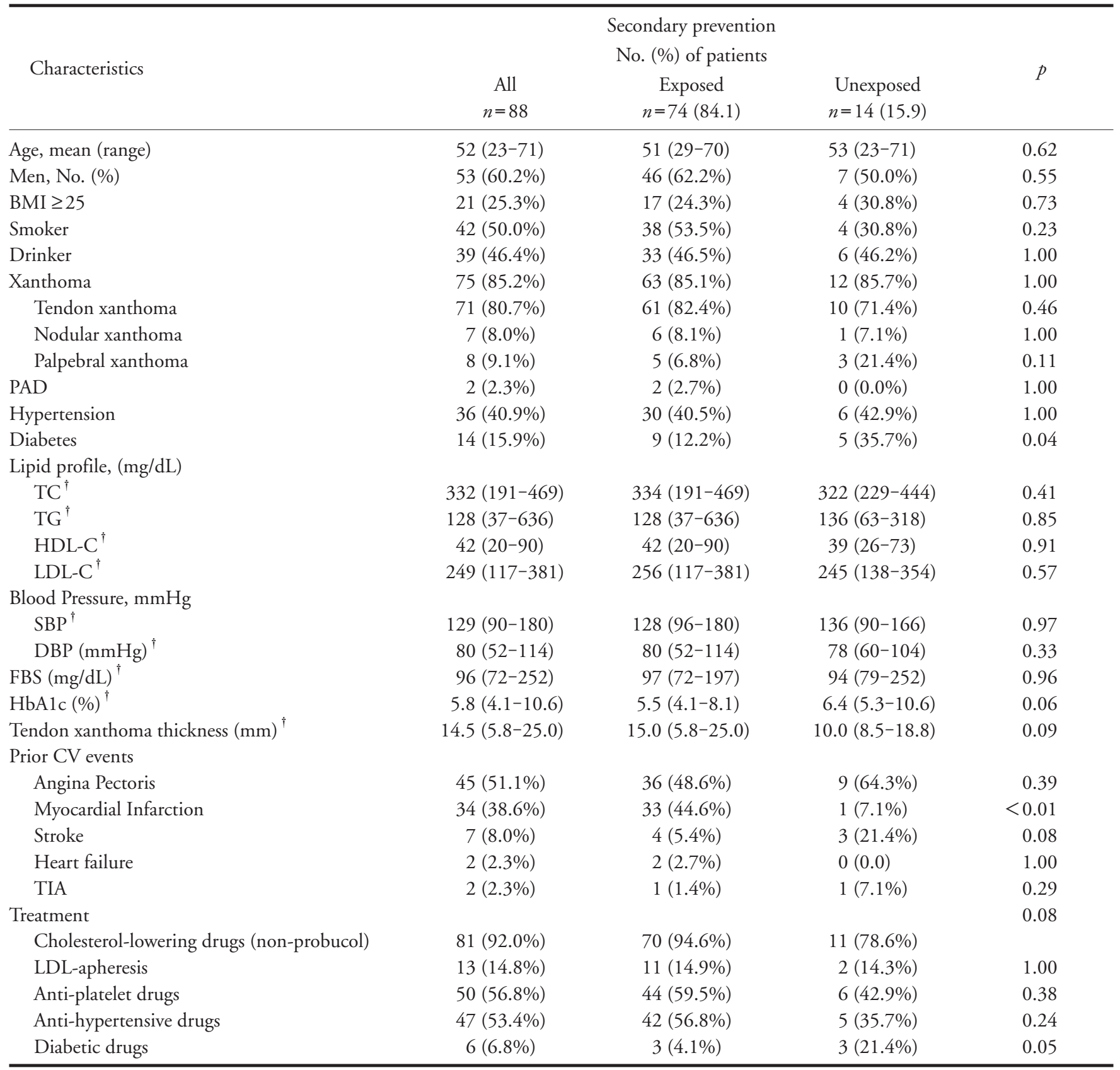

\footnotetext{
${ }^{\dagger}$ Data are the median (range). All data are numbers (\%) unless otherwise indicated. Each percentage is related to the total number with measurement data. TIA indicates transient ischemic attack.
}

147 (124-197) and 33 (17-70) mg/dL. Sub-analysis of changes in the lipid profile after probucol treatment detected significant three predictors of $\mathrm{CV}$ event risk: higher baseline TC (HR 2.74, 95\% CI 1.05-7.16; $p=$ $0.04)$ in the primary prevention group; reduction in TG (HR 0.22, 95\% CI 0.06-0.86; $p=0.03$ ); and reduction in LDL-C (HR 0.17,95\% CI 0.03-0.90; $p=0.04)$ after treatment in the subset of the secondary prevention group on stable doses of probucol. Neither TC nor HDL-C after treatment was associated with $\mathrm{CV}$ event risk in the probucol-exposed group, which indicates that reduction of the HDL-C level after probucol treatment is not related to $\mathrm{CV}$ event risk for probucol-exposed patients.

We evaluated the safety of probucol for all collected data from 541 patients, and found 56 adverse 
Table 3. Incidence of cardiovascular events

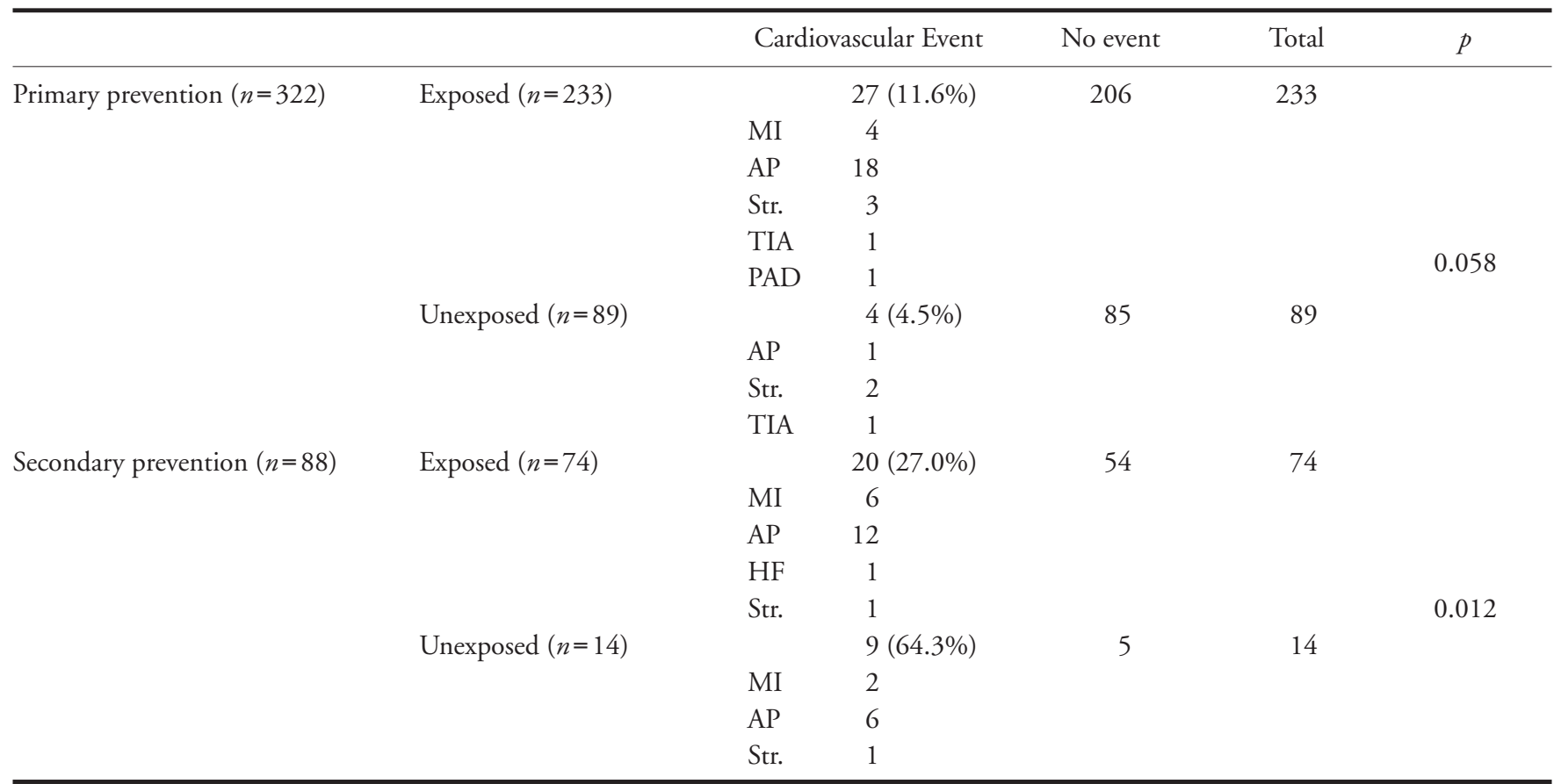

MI, myocardial infarction; AP, angina pectoris; HF, heart failure; Str., stroke; TIA, transient ischemic attack; PAD, peripheral artery disease.

One of the 4 patients died after 12 months of probucol termination.

events in 18 patients. Malaise, pruritus, macrocytic anemia and pain in the extremities were recorded as adverse drug reactions associated with probucol. We noted and reported gastric cancer stage III immediately to the Ministry of Health and Welfare as an unexpected serious event, because of an unknown drug relation due to many concomitant drugs, although probucol was found to be non-carcinogenic alone ${ }^{21)}$. Six deaths were observed in the population not taking probucol or stopping probucol. There was no other difference in the incidence of adverse events, including serious events, between probucol exposure and non-exposure.

\section{Discussion}

Many data from large-scale randomized controlled trials have overwhelmingly demonstrated the clinical benefits of lowering cholesterol with statins ${ }^{22,23)}$, yet the rapid and extensive prophylactic use of cholesterol-lowering drugs remains controversial. Few studies have addressed the clinical risks and benefits of longterm treatment of hyperlipidemia among women ${ }^{24)}$ or elderly patients ${ }^{25)}$. The safety of long-term cholesterollowering therapy, including the issue of associated cancer risk or benefit, remains inconclusive because of conflicting clinical evidence ${ }^{26}$. More importantly, conclusions from the results of randomized controlled trials are limited by their relatively short follow-up periods (generally less than 5 years) in the analyzed studies.

In long-term treatment for $\mathrm{FH}$, probucol was used with other cholesterol lowering drugs in over $80 \%$ of the secondary prevention group-those with a more severe clinical outlook than the primary prevention group: a higher prevalence of hypertension and diabetes, significant thicker tendon xanthoma, more combined therapy with LDL-apheresis, anti-platelet drugs, and anti-hypertensive drugs. The high rate of probucol use in FH was surprising, different from expected. This might partly reflect the prescription behavior of experts with the result that intractable patients responded to the regimen.

In the secondary prevention, the higher-risk group, probucol exposure was associated with a reduction in the risk of cardiovascular events (HR 0.13; 95\% CI $0.05-0.34)$ with high significance $(p<0.001)$, while it was not significant in the primary prevention group. This result was also contrary to our expectation that probucol exposure would likely be associated with increased event risk due to a confounding indication-that patients considered more severe at diagnosis would receive more treatment, including probucol. We did not collect the details of non-probucol drugs 


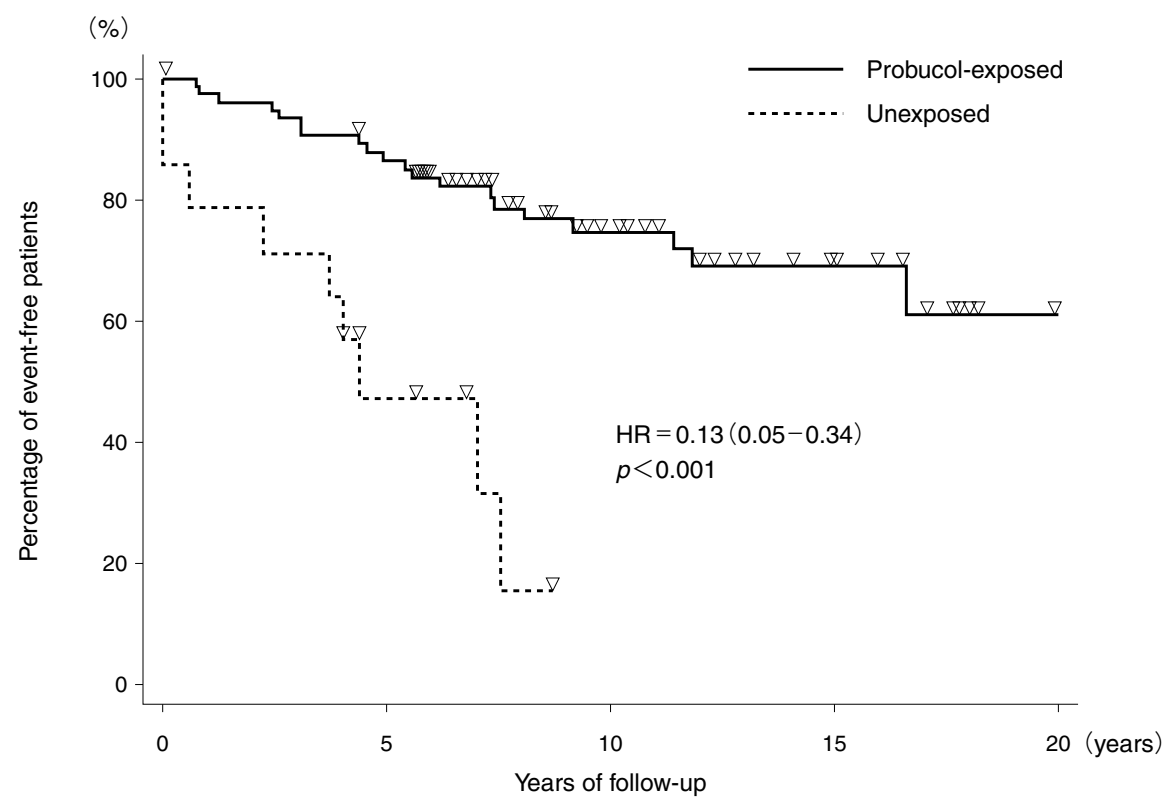

\begin{tabular}{|c|c|c|c|c|c|c|c|c|c|c|c|c|c|c|c|c|c|c|c|c|c|}
\hline \multicolumn{22}{|c|}{ Number at risk } \\
\hline Years & 0 & & & & & 5 & & & & & 10 & & & & & 15 & & & & & 20 \\
\hline Exposed & 74 & 71 & 70 & 68 & 66 & 62 & 54 & 50 & 42 & 38 & 34 & 30 & 25 & 19 & 17 & 13 & 12 & 8 & 3 & 1 & 0 \\
\hline Unexposed & 14 & 11 & 11 & 10 & 9 & 5 & 4 & 2 & 1 & 0 & 0 & 0 & 0 & 0 & 0 & 0 & 0 & 0 & 0 & 0 & 0 \\
\hline
\end{tabular}

Estimates of event-free rates are according to whether patients received probucol. The cumulative probability of remaining without events was higher in patients treated with probucol ( $p<0.001$; log-rank test).

Fig. 2. Kaplan-Meier Estimates of Event-free Rate.

For secondary prevention, the incidence of cardiovascular events was $27.0 \%$ in the exposed group and $64.3 \%$ in the unexposed group. An event-free survival curve for the secondary prevention group is given.

Table 4. The results of multivariate analysis using Cox regression procedure

\begin{tabular}{|c|c|c|c|c|c|c|}
\hline \multirow{2}{*}{ Factor } & \multicolumn{3}{|c|}{ Primary prevention } & \multicolumn{3}{|c|}{ Secondary prevention } \\
\hline & $\mathrm{HR}$ & $95 \% \mathrm{CI}$ & $p$ & HR & $95 \% \mathrm{CI}$ & $p$ \\
\hline \multicolumn{7}{|l|}{ Baseline variables } \\
\hline Total cholesterol & 1.58 & $1.06-2.33$ & 0.02 & - & - & - \\
\hline Drinking & 2.43 & $1.09-5.44$ & 0.03 & - & - & - \\
\hline Peripheral artery disease & 5.27 & $0.51-54.63$ & 0.16 & - & - & - \\
\hline Palpebral xanthoma & - & - & - & 2.94 & $1.02-8.47$ & 0.05 \\
\hline Diabetes & - & - & - & 2.58 & $0.76-8.76$ & 0.13 \\
\hline \multicolumn{7}{|l|}{ Treatment in follow-up } \\
\hline Probucol use & 1.50 & $0.48-4.67$ & 0.49 & 0.13 & $0.05-0.34$ & $<0.001$ \\
\hline Anti-platelet drug use & - & - & - & 2.48 & $1.00-6.17$ & 0.05 \\
\hline
\end{tabular}

to simplify the study procedure. However, we would likely exclude underused statins because of the reduced use of non-probucol drugs from the possible factors of the higher event rate in the unexposed group, because statins were available when all of the 9 recurrent patients (Table 3) started and the patients continued on cholesterol-lowering drugs. We suppose, therefore, that the reasons for this unanticipated great risk reduction include some antioxidant and anti-atherogenic actions $^{3,4,27)}$ of probucol. The finding in second prevention may be suggested by the report ${ }^{27)}$ that probucol significantly decreased in vitro LDL oxidizability measured under typically strong oxidative conditions, and that long-term treatment with probucol had an 
anti-atherogenic effect in Watanabe Heritable Hyperlipidemic rabbits. From the observation that the baseline lipid profile was not different between the two groups of exposure and non-exposure in secondary prevention, the drug might exhibit greates effectiveness in post-cardiovascular disease patients, in possibly advanced lipid accumulation and inflammation, which are associated with the circulation of oxidized $\mathrm{LDL}^{28)}$.

In primary prevention, we observed an almost significant increase of events in the exposed group (Table 3), and an apparently increased risk (HR 1.5), although not statistically significant after adjustment (Table 4). We suppose, however, that the ideal effects of probucol might be concealed by the following factors noted in primary prevention. The exposed group had a worse lipid profile (TC, LDL-C and HDL-C levels), higher $\mathrm{HbA}_{1 \mathrm{c}}$, and thus definitely a higher risk than the unexposed group. Furthermore, 8 (nearly $30 \%$ ) of the 27 patients experiencing cardiovascular events in the exposed group discontinued probucol when they had events. This was consistent with the different finding between primary and secondary preventions in the exposed group: less than half of the patients (113 of 233) in primary prevention continued on probucol, while $53(72 \%)$ of 74 patients continued in secondary prevention. This estimation might be conservative.

The controversial and paradoxical action of probucol- lowering HDL-C- level was not associated with the risk of $\mathrm{CV}$ events in the cohort, therefore, the association between low levels of HDL-C and an increased risk for $\mathrm{CV}$ events or death indicated by the early Framingham Heart Study ${ }^{29)}$ may not be extrapolated to probucol-treated patients. This proposition is consistent with recent findings that a lowered HDL-C level is not always atherogenic, but that the quality or function of HDL-C is more important than the HDL-C levels ${ }^{30)}$. In fact, increased levels of HDL-C with torcetrapib, a CETP inhibitor, were not associated with a significant clinical benefit in patients with coronary disease ${ }^{31)}, \mathrm{FH}^{32)}$ or mixed dyslipidemia ${ }^{33)}$.

We speculate that enhanced reverse cholesterol transport by CETP activation as a result of probucol treatment also contributed to the detected risk reduction in the cohort. The observed positive outcome of probucol, a CETP activator, might be a mirror image of the negative clinical trial results for the CETP inhibitor $^{34)}$. Reports ${ }^{35,36)}$ of increased coronary heart disease in CETP deficiency despite increased HDL-C levels, and the molecular approach to review CETP deficiency ${ }^{37)}$ support our hypothesis, at least in Japanese genealogy. Interestingly, a recent basic research reports that human CETP expression enhances the mouse survival rate in an experimental systemic inflammation model ${ }^{38)}$, indicating for the first time a role for CETP in the defense against the exacerbated production of proinflammatory mediators.

For the safety evaluation, we found no cardiotoxic adverse drug reaction including QT/QTc prolongation or torsade de pointes, in this study, although probucol can cause them ${ }^{16,39,40)}$.

We obtained these results from an observational study with no control for inaccuracy, unexpected bias or confounding factors. We could not assure the precision of the baseline measurements due to unrecorded data. The participant centers were major hospitals for $\mathrm{FH}$, but not all hospitals in Japan, because the study was conducted as part of a post-marketing study by a pharmaceutical manufacturer within the framework of the Japanese government regulations. Some restrictions on collecting data might have resulted in unexpected small numbers in the unexposed group in secondary prevention, although we think that the study cohort represents nearly a nationwide population of heterozygous FH in Japan. The results derived from patient data in Japan can not necessarily be generalized to patients in western countries.

Despite these limitations of the study, however, we could evaluate the outcome of long-term probucol treatment in the medical practice setting for $\mathrm{FH}$, a high-risk population, for as long as 20 years in Japan. The significant risk reduction of $\mathrm{CV}$ events observed in the secondary prevention group holds clinical significance and suggests some beneficial therapeutic actions of this drug in arteriosclerotic diseases. The hypothesis from the findings warrants a randomized controlled trial for verification of the secondary prevention, and needs further research into the molecular mechanisms or roles of CETP in pathogenesis.

\section{Author Contributions}

Dr. Yamashita had full access to all of the data in the study and takes responsibility for the integrity of the data and the accuracy of the data analysis. Study concept and design: Matsuzawa, Kita, Saito, Fukushima, Matsui. Acquisition of data: Yamashita, Bujo, Arai, Harada-Shiba, Saito, Kita, Matsuzawa. Analysis and interpretation of data: Yamashita, Bujo, Arai, Harada-Shiba, Matsui, Saito, Fukushima, Kita, Matsuzawa.

Drafting of the manuscript: Yamashita, Bujo, Arai, Harada-Shiba, Matsui, and Fukushima. Critical revision of the manuscript for important intellectual content: Yamashita, Matsui, Fukushima, Kita, Saito, 
and Matsuzawa. Statistical analysis: Matsui and Fukushima. Administrative, technical, or material support: Fukushima, Matsui, Kita, Saito, and Matsuzawa. Study supervision: Yamashita, Fukushima, Matsui, Kita, Saito, and Matsuzawa.

\section{Acknowledgements}

We thank all the study participants, POSITIVE investigators, and the sponsor co-workers' assistance; $\mathrm{T}$. Yoshida for medical writing, Y. Okutani and H. Doi for coordination and management of the study organization.

\section{Sources of Funding}

Daiichi Sankyo Co., Ltd. (formerly Daiichi) and Otsuka Pharmaceutical Co., Ltd. (Otsuka) sponsored and jointly conducted the study in compliance with the legislated procedure of Good Post-marketing Study Practice toward the drug re-evaluation system under the Ministry of Health and Welfare in Japan.

\section{Disclosures}

From the formerly Daiichi and Otsuka, Dr. Matsui, Dr. Fukushima, Dr. Matsuzawa, and Dr. Kita received fees and expenses for meetings related to protocol design, statistical and clinical interpretation of the data; Dr. Bujo, Dr. Arai, Dr. Harada-Shiba received honoraria and travel expenses for lectures, Dr.Yamashita, Dr. Bujo, Dr. Arai received fees and travel expenses for a meeting related to clinical interpretation of the data. Dr. Yamashita received consultancy fees from Otsuka. Dr. Matsuzawa is contracted as a short-term adviser to Otsuka in medical science. Dr. Saito received travel expenses only.

\section{POSITIVE Investigators}

Osaka University Hospital, Suita (S. Yamashita, T. Maruyama); National Cardiovascular Center, Suita (M. Harada-Shiba); Sumitomo Hospital, Osaka (Y. Minami); Chiba University Hospital, Chiba (H. Bujo); Asahi General Hospital, Asahi (N. Hashimoto); Kawatetsu Chiba Hospital, Chiba (M. Takahashi); Nishifuna Naika, Funabashi (M. Shinomiya); Kashiwado Hospital, Chiba (K. Kosuge); Numazu City Hospital, Numazu (Y. Hayashi); Toho University Sakura Medical Center, Sakura (K. Shirai, Y. Miyashita); Matsudo City Hospital, Matsudo (T. Oeda); Kyoto University Hospital, Kyoto (M.Yokode, H. Arai); Hiroshima General Hospital of West Japan Railway Company,
Hiroshima (K. Takata); Maizuru Kyosai Hospital, Maizuru (R. Tatami); Kido Hospital, Niigata (T. Miida)

\section{References}

1) Barnhart JW, Sefranka JA, McIntosh DD: Hypocholesterolemic effect of 4,4'-(isopropylidenedithio)-bis (2,6-di-tbutylphenol) (probucol). Am J Clin Nutr, 1970; 23:12291233

2) Tawara K, Tomikawa M, Abiko Y: Mode of action of probucol in reducing serum cholesterol in mice. Jpn J Pharmacol, 1986; 40:123-133

3) Kita T, Nagano Y, Yokode M, Ishii K, Kume N, Ooshima A, Yoshida H, Kawai C: Probucol prevents the progression of atherosclerosis in Watanabe heritable hyperlipidemic rabbit, an animal model for familial hypercholesterolemia. Proc Natl Acad Sci USA, 1987; 84:5928-5931

4) Carew TE, Schwenke DC, Steinberg D: Antiatherogenic effect of probucol unrelated to its hypocholesterolemic effect: evidence that antioxidants in vivo can selectively inhibit low density lipoprotein degradation in macrophage-rich fatty streaks and slow the progression of atherosclerosis in the Watanabe heritable hyperlipidemic rabbit. Proc Natl Acad Sci USA, 1987; 84:7725-7729

5) Siveski-Iliskovic N, Kaul N, Singal PK: Probucol promotes endogenous antioxidants and provides protection against adriamycin-induced cardiomyopathy in rats. Circulation, 1994; 89:2829-2835

6) McPherson R, Hogue M, Milne RW, Tall AR, Marcel YL: Increase in plasma cholesteryl ester transfer protein during probucol treatment. Relation to changes in high density lipoprotein composition. Arterioscler Thromb, 1991; 11:476-481

7) Chiesa G, Michelagnoli S, Cassinotti M, Gianfranceschi G, Werba JP, Pazzucconi F, Sirtori CR, Franceschini G: Mechanisms of high-density lipoprotein reduction after probucol treatment: changes in plasma cholesterol esterification/transfer and lipase activities. Metabolism, 1993; 42:229-235

8) Ishigami $M$, Yamashita $S$, Sakai N, Hirano $K$, Arai T, Maruyama T, Takami S, Koyama M, Kameda-Takemura K, Matsuzawa Y: High-density lipoproteins from probucol-treated patients have increased capacity to promote cholesterol efflux from mouse peritoneal macrophages loaded with acetylated low-density lipoproteins. Eur J Clin Invest, 1997; 27:285-292

9) Rinninger F, Wang N, Ramakrishnan R, Jiang XC, Tall AR: Probucol enhances selective uptake of HDL-associated cholesteryl esters in vitro by a scavenger receptor B-Idependent mechanism. Arterioscler Thromb Vasc Biol, 1999; 19:1325-1332

10) Hirano K, Ikegami C, Tsujii K, Zhang Z, Matsuura F, Nakagawa-Toyama Y, Koseki M, Masuda D, Maruyama T, Shimomura I, Ueda Y, Yamashita S: Probucol enhances the expression of human hepatic scavenger receptor class B type 1, possibly through a species-specific mechanism. Arterioscler Thromb Vasc Biol, 2005; 25:2422-2427

11) Matsuzawa $Y$, Yamashita $S$, Funahashi $T$, Yamamoto $A$, Tarui S: Selective reduction of cholesterol in HDL2 frac- 
tion by probucol in familial hypercholesterolemia and hyper HDL2 cholesterolemia with abnormal cholesteryl ester transfer. Am J Cardiol, 1988; 62:66B-72B

12) Yamamoto A, Matsuzawa $Y$, Yokoyama $S$, Funahashi $T$, Yamamura T, Kishino B: Effects of probucol on xanthomata regression in familial hypercholesterolemia. Am J Cardiol, 1986; 57:29H-35H

13) Shinomiya M, Nishide T, Tashiro J, Shirai K, Saito Y, Yoshida S: Effect of 5-year administration of probucol on development of myocardial infarction in heterozygous familial hypercholesterolemia. Current Ther Res, 1993; 54:142-151

14) Daida H, Kuwabara Y, Yokoi H, Nishikawa H, Takatsu F, Nakata Y, Kutsumi Y, Oshima S, Nishiyama S, Ishiwata S, Kato K, Nishimura S, Miyauchi K, Kanoh T, Yamaguchi $\mathrm{H}$ : Effect of probucol on repeat revascularization rate after percutaneous transluminal coronary angioplasty (from the Probucol Angioplasty Restenosis Trial [PART]). Am J Cardiol, 2000; 86:550-552, A9

15) Tardif JC, Cöté G, Lespérance J, Bourassa M, Lambert J, Doucet S, Bilodeau L, Nattel S, de Guise P: Probucol and multivitamins in the prevention of restenosis after coronary angioplasty. Multivitamins and Probucol Study Group. N Engl J Med, 1997; 337:365-372

16) Tardif JC, Grégoire J, Schwartz L, Title L, Laramée L, Reeves F, Lespérance J, Bourassa MG, L'Allier PL, Glass M, Lambert J, Guertin MC; Canadian Antioxidant Restenosis Trial (CART-1) Investigators: Effects of AGI-1067 and probucol after percutaneous coronary interventions. Circulation, 2003; 107:552-558

17) Baldassarre D, Franceschini G, Peruzzotti G, Brusoni B, Sirtori CR: Clinical evaluation of probucol in hypercholesterolemia: individual lipoprotein responses and inhibitory effect on carotid atherosclerosis progression. J Cardiovasc Pharmacol, 1997; 30:784-789

18) Sawayama Y, Shimizu C, Maeda N, Tatsukawa M, Kinukawa N, Koyanagi S, Kashiwagi S, Hayashi J: Effects of probucol and pravastatin on common carotid atherosclerosis in patients with asymptomatic hypercholesterolemia. Fukuoka Atherosclerosis Trial (FAST). J Am Coll Cardiol, 2002; 39:610-616

19) Teramoto T, Sasaki J, Ueshima H, Egusa G, Kinoshita M, Shimamoto K, Daida H, Biro S, Hirobe K, Funahashi T, Yokote K, Yokode M: Committee for Epidemiology and Clinical Management of Atherosclerosis: Primary hyperlipidemia. J Atheroscler Thromb, 2008; 15:49-51

20) DeLong DM, DeLong ER, Wood PD, Lippel K, Rifkind BM: A comparison of methods for the estimation of plasma low- and very low-density lipoprotein cholesterol. The Lipid Research Clinics Prevalence Study. JAMA, 1986; 256:2372-2377

21) Newman TB, Hulley SB: Carcinogeniciy of lipid-lowering drugs. JAMA, 1996; 275:55-60

22) Cholesterol Treatment Trialists' (CTT) Collaborators: Efficacy and safety of cholesterol-lowering treatment: prospective meta-analysis of data from 90056 participants in 14 randomised trilas of statins. Lancet, 2005; 366:12671278

23) Nakamura H, Arakawa K, Itakura H, Kitabatake A, Goto Y, Toyota T, Nakaya N, Nishimoto S, Muranaka M,
Yamamoto A, Mizuno K, Ohashi Y; MEGA Study Group: Primary prevention of cardiovascular disease with pravastatin in Japan (MEGA Study): a prospective randomised controlled trial. Lancet, 2006; 368:1155-1163

24) Walsh JM, Pignone M: Drug treatment of hyperlipidemia in women. JAMA, 2004; 291:2243-2252

25) Ali R, Alexander KP: Statins for the primary prevention of cardiovascular events in older adults: a review of the evidence. Am J Geriatr Pharmacother, 2007; 5:52-63

26) Bonovas S, Filioussi K, Tsavaris N, Sitaras NM: Statins and cancer risk: a literature-based meta-analysis and metaregression analysis of 35 randomized controlled trials. J Clin Oncol, 2006; 24:4808-4817

27) Bräsen JH, Koenig K, Bach $H$, Kontush $A$, Heinle $H$, Witting PK, Ylä-Herttuala S, Stocker R, Beisiegel U: Comparison of the effects of alpha-tocopherol, ubiquinone-10 and probucol at therapeutic doses on atherosclerosis in WHHL rabbits. Atherosclerosis, 2002; 163:249259

28) Tsimikas S, Brilakis ES, Miller ER, McConnell JP, Lennon RJ, Kornman KS, Witztum JL, Berger PB: Oxidized phospholipids, $\mathrm{Lp}(\mathrm{a})$ lipoprotein, and coronary artery disease. N Engl J Med, 2005; 353:46-57

29) Wilson PW, Abbott RD, Castelli WP: High density lipoprotein cholesterol and mortality. The Framingham Heart Study. Arteriosclerosis, 1988; 8:737-741

30) Navab M, Anantharamaiah GM, Reddy ST, Van Lenten BJ, Ansell BJ, Fogelman AM: Mechanisms of disease: proatherogenic HDL--an evolving field. Nat Clin Pract Endocrinol Metab, 2006; 2:504-511

31) Nissen SE, Tardif JC, Nicholls SJ, Revkin JH, Shear CL, Duggan WT, Ruzyllo W, Bachinsky WB, Lasala GP, Tuzcu EM; ILLUSTRATE Investigators. Effect of torcetrapib on the progression of coronary atherosclerosis. $\mathrm{N}$ Engl J Med, 2007; 356:1304-1316

32) Kastelein JJ, van Leuven SI, Burgess L, Evans GW, Kuivenhoven JA, Barter PJ, Revkin JH, Grobbee DE, Riley WA, Shear CL, Duggan WT, Bots ML; RADIANCE 1 Investigators. Effect of torcetrapib on carotid atherosclerosis in familial hypercholesterolemia. N Engl J Med, 2007; 356:1620-1630

33) Bots ML, Visseren FL, Evans GW, Riley WA, Revkin JH, Tegeler CH, Shear CL, Duggan WT, Vicari RM, Grobbee DE, Kastelein JJ; RADIANCE 2 investigators. Torcetrapib and carotid intima-media thickness in mixed dyslipidaemia (RADIANCE 2 study): a randomized, doubleblind trial. Lancet, 2007; 370:153-160

34) Barter PJ, Caulfield M, Eriksson M, Grundy SM, Kastelein JJ, Komajda M, Lopez-Sendon J, Mosca L, Tardif JC, Waters DD, Shear CL, Revkin JH, Buhr KA, Fisher MR, Tall AR, Brewer B; ILLUMINATE Investigators: Effects of torcetrapib in patients at high risk for coronary events. N Engl J Med, 2007; 357:2109-2122

35) Zhong S, Sharp DS, Grove JS, Bruce C, Yano K, Curb JD, Tall AR: Increased coronary heart disease in JapaneseAmerican men with mutation in the cholesteryl ester transfer protein gene despite increased HDL levels. J Clin Invest 1996; 97:2917-2923

36) Hirano K, Yamashita S, Nakajima N, Arai T, Maruyama T, Yoshida Y, Ishigami M, Sakai N, Kameda-Takemura K, 
Matsuzawa Y: Genetic cholesteryl ester transfer protein deficiency is extremely frequent in the Omagari area of Japan. Marked hyperalphalipoproteinemia caused by CETP gene mutation is not associated with longevity. Arterioscler Thromb Vasc Biol, 1997; 17:1053-1059

37) Nagano M, Yamashita S, Hirano K, Takano M, Maruyama T, Ishihara M, Sagehashi Y, Kujiraoka T, Tanaka K, Hattori H, Sakai N, Nakajima N, Egashira T, Matsuzawa Y: Molecular mechanisms of cholesteryl ester transfer protein deficiency in Japanese. J Atheroscler Thromb, 2004; 11:110-121

38) Cazita PM, Barbeiro DF, Moretti AI, Quintão EC, Sori- ano FG: Human cholesteryl ester transfer protein expression enhances the mouse survival rate in an experimental systemic inflammation model: a novel role for CETP. Shock, 2008; 30:590-595

39) Viskin S, Justo D, Halkin A, Zeltser D: Long QT syndrome caused by noncardiac drugs. Prog Cardiovasc Dis, 2003; 45:415-427

40) Guo J, Massaeli H, Li W, Xu J, Luo T, Shaw J, Kirshenbaum LA, Zhang S. Identification of IKr and its trafficking disruption induced by probucol in cultured neonatal rat cardiomyocytes. J Pharmacol Exp Ther, 2007; 321:911920 